\title{
Gene Expression of Indoleamine 2,3 Dioxygenase 1, Insulin-Growth Factor 1 and Red/IK Cytokine in Alopecia Areata
}

\author{
Simona ȘENILĂ ${ }^{1}$, Ovidiu BĂLĂCESCU², Loredana BĂLĂCESCU², \\ Elisabeta CANDREA ${ }^{1}$, Loredana UNGUREANU ${ }^{1}$, \\ Sorina DĂNESCU ${ }^{1}$, Rodica COSGAREA ${ }^{1 *}$ \\ ${ }^{1}$ University of Medicine and Pharmacy "Iuliu Hațieganu", Department of Dermatology, 3-5 Clinicilor, Cluj-Napoca, Romania \\ ${ }^{2}$ Functional Genomics, Proteomics and Experimental Pathology Laboratory, Institute of Oncology "Ion Chiricuță", 34-36 Republicii, \\ Cluj-Napoca, Romania; rocosgarea@gmail.com (*corresponding author)
}

\begin{abstract}
Alopecia areata (AA) is a chronic, T-cell mediated autoimmune disease directed against the hair follicle, which partially evolves due to a loss of the immune privilege of the anagen hair follicle. The immune privilege is maintained by several factors, including a downregulation of MHC class I and II, local immunosupressants and expression of Fas ligand. The purpose of the study was to evaluate several factors involved in the collapse and restoration of the immune privilege. We investigated IDO1, IGF1 and red/IK gene expression in lesional and perilesionalscalp biopsies from alopecia areata patients. Seven paired punch-biopsies were taken from the active edge of alopecic plaque and from the perilesional scalp. Expression of IDO1, IGF1 and red/IK genes was performed by qRT-PCR. In lesional tissue, IGF1, IDO1 and red/IK genes showed an increase in the mRNA levels as compared with the perilesional scalp. By comparing the pairs of data for the investigated genes, IDO1was statistically upregulated in the lesional area. No significant differences were observed between the gene expression in mild or severe AA, from the lesional or perilesional areas. IDO1 mRNA expression was higher in patients with a relapse duration of less than 6 months as compared to patients with a relapse duration of more than 6 months; levels of IGF1 and red/IK mRNA are increased in lesionals compared to perilesional scalp area.
\end{abstract}

Keywords: hair loss, immune privilege, restoration

\section{Introduction}

Alopecia areata $(\mathrm{AA})$ is a chronic, $\mathrm{T}$-cell mediated autoimmune disease directed against the hair follicle. It is characterized by patchy hair loss on the scalp or body (including eyebrows and eyelashes) with an unpredictable evolution. Even though significant progress was made in the pathogenesis of AA, the cascade of events is not completely understood. It has been demonstrated a relationship between AA and genetic predisposition (specific HLA class II alleles), autoantigens, lymphocytic accumulation around the hair follicle ("swarm of bees"), NKG2D expression in NK cells, CD8+, gamma delta T cells and stress hormone (Ito 2013). Alongside the anterior chamber of the eye, the central nervous system (behind the brain-blood barrier), placenta and testis, the hair follicle benefits of an immune privilege, a mechanism in which several factors collaborate in order to suppress a cytotoxic immune attack against cells and antigens (Paus et al., 2003). Of several hypotheses for AA pathogenesis, one suggests that AA is a consequence of immune privilege collapse (Ito et al., 2004). Patients with a specific genetic background are susceptible to developing AA, most probably by downregulation of the immunosuppressive environment. This process involves cytokines which downregulate MHC class I (insulin growth factor 1-IGF1) and class II (red/IK cytokine) antigens expression and local immunosupressive molecules which are induced by IFN-gamma such as Indoleamine 2,3dioxygenase 1(IDO1)(Gilhar 2010). Our aim was to evaluate the gene expression of these factors (IDO1, IGF1 and red/IK)involved in the collapse or restoration of the immune privilege in alopecia areata patients.

\section{Materials and methods}

\section{Patients and tissue samples}

Seven patients ( 3 females and 4 males with mean age of 25.86 years) with plurifocal, active AA: two patients with mild form of AA (less than $10 \%$ scalp affected) and five patients with severe form of AA (more than $40 \%$ scalp affected) were included in this analytic, cross sectional study. The patients were informed about the study procedure and 
264

gave their written informed consent in accordance with the ethical committee approval of the Emergency County Hospital Cluj-Napoca, Romania.

The study was performed between 2010-2012 at the Department of Dermatology of the University of Medicine and Pharmacy "Iuliu Haţieganu" Cluj-Napoca and Functional Genomics, Proteomics and Experimental Pathology Laboratory of the Institute of Oncology "Ion Chiricuță” Cluj-Napoca.

Two punch biopsies, 3-mm each, were performed as following: the first biopsy was taken from the active edge of $\mathrm{AA}$ and the second-from a clinically non-affected area, at a 2 $\mathrm{cm}$ distance from first biopsy, where the pull test was negative. Eleven control scalp specimens were obtained from surgery subjects. Tissues were stored in liquid nitrogen at a temperature of $-80^{\circ} \mathrm{C}$. The level of expression for three genes (IDO1, IGF1 and red/IK) in lesional and perilesional areas of AA patients and controls have been evaluated by qRT-PCR.

\section{Quantitative Reverse Transcriptase-Polymerase Chain Reaction ( $q R T-P C R$ )}

RNA was extracted using Tri Reagent ${ }^{\circ}$ (Sigma-Aldrich) according to the manufacturer's instructions by phenolchloroform method and was purified with silica-membrane spin columns from the RNeasy mini kit (Qiagen). The quantity of the RNA was quantified with NanoDrop ND1000 spectrophotometer and the quality was assessed by Agilent 2100 Bioanalyzer 2100 Instrument. A FirstStrand cDNA Synthesis Kit (Roche) was used to reverse transcribe $200 \mathrm{ng}$ of total RNA. Five microliters of 1:10 (v/v)-diluted cDNA was amplified in a final volume of $20 \mu \mathrm{l}$ using a LightCycler 480 (Roche). The amplification was performed with $1 \mu \mathrm{M}$ specific primers (Tip Molbiol) and a $0.2 \mu \mathrm{M}$ specific hydrolysis probe from the Universal Probe Library (UPL). The primers and UPL probes were designed with Roche Applied Science software as follows: IDO1 (NM_002164.5): F-ttcag tgctttgacgtcctg, Ratgtcctggaggaactgagc, (UPL\#22); red/IK(NM_006083.3): F-agctgacccagatcctttca, R-tcagcctcaggaggtttctt, (UPL\# 11); IGF1(NM_001111283.1): F-tggagacaggggcttttattt, Raggggtgcgcaatacatc, (UPL\#75). The qRT-PCR evaluation of each gene was performed in triplicate. Gene expression levels were calculated by ddct method after using the housekeeping gene 18rRNA. The ddct method, a convenient method to analyze the relative changes in gene expression, requires the assignment of one or more housekeeping genes, which are assumed to be uniformly and constantly expressed in all samples, as well as in one or more reference samples. The expression of other samples is then compared to that in the reference sample (Livak et al., 2001). Fold change (FC) values were calculated according to gene expression values for the three genes from the lesional and perilesional areas comparing it to the mean value of each gene from the control group. The genes with
FC $>1.3$ and $p<0.05$ were considered as significantly differentially expressed between studied groups.

\section{Statistical analysis}

Qualitative variables were summarized as percentages while quantitative variables were summarised as mean and standard deviations for normally distributed data. Comparisons between groups were done with $\mathrm{Z}$ test for qualitative variables and paired samples t-test (lesional and perilesional) for quantitative variables. Statistical analysis was conducted with Statistica (v.8) program at a significance level of $5 \%$. Graphical representations were done with Microsoft Excel.

\section{Results}

In lesional AA tissue, IGF1, IDO1 and red/IK genes showed increase in the mRNA levels compared with the perilesional AA. Mean fold change values and standard deviation for investigated genes and the result of comparison between pairs of data are presented in Tab. 1 .

By comparing the pairs of data for the investigated genes, only IDO1 reached statistical significance $(p=0.04)$, demonstrating the fact that this gene is upregulated $(\mathrm{FC}=1.86)$ in the lesional area (Fig. 1).

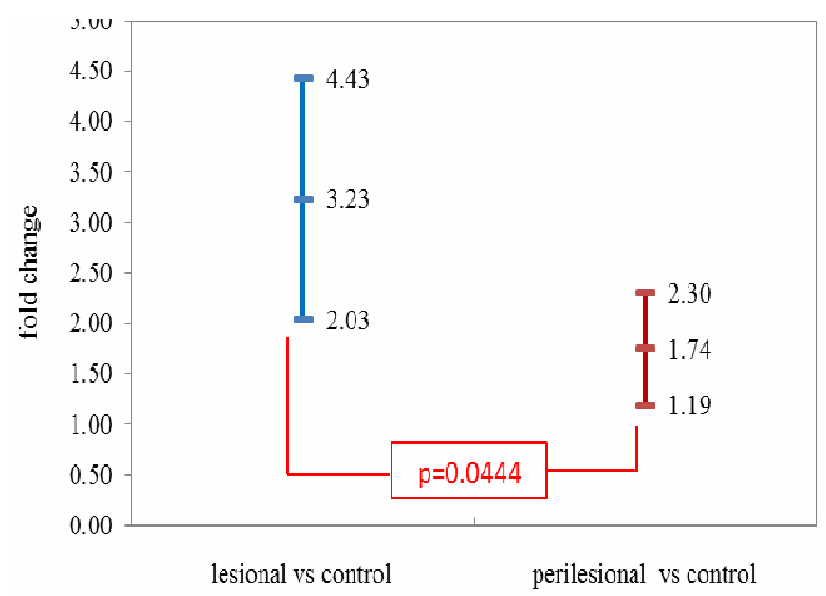

Fig. 1. Mean fold change values for IDO1 in lesional and perilesional areas ( $95 \%$ CI values of FC)

Two out of seven patients $(28.57 \%)$ had mild alopecia areata and all other $(71.43 \%)$ had severe alopecia areata. The proportion of patients with severe alopecia areata proved significantly higher than the proportion of patients with mild alopecia areata (Z-statistics $=2.5102$, p-value $=$ $0.0121)$. Two patients with severe form of AA (28.57\%) had the disease for more than one year and two patients with severe alopecia had the relapse duration for more than 6 months (28.57\%), this proportion being significantly smaller compared to proportion of patients who had the

Tab.1. Mean fold change (FC) and standard deviation values for IGF1, IDO1 and red/IK genes

\begin{tabular}{|c|c|c|c|c|c|c|}
\hline \multirow{2}{*}{ Variable } & \multicolumn{2}{|c|}{ FC lesional vs control } & \multicolumn{2}{|c|}{ FC perilesional vs control } & \multirow{2}{*}{$\begin{array}{c}\mathrm{t} \text {-statistics (p-value) } \\
\mathrm{m} \pm \text { stdev }\end{array}$} & \multirow{2}{*}{$\begin{array}{c}\text { FC lesional vs } \\
\text { perilesional }\end{array}$} \\
\hline & $\mathrm{m} \pm$ stdev & $(\min -\max )$ & $\mathrm{m} \pm$ stdev & $(\min -\max )$ & & \\
\hline IGF1 & $2.41 \pm 2.61$ & $(0.73-7.98)$ & $1.83 \pm 1.27$ & $(0.61-4.22)$ & IGF1 & $2.41 \pm 2.61$ \\
\hline IDO1 & $3.23 \pm 1.29$ & $(1.44-4.91)$ & $1.74 \pm 0.60$ & $(0.67-2.68)$ & IDO1 & $3.23 \pm 1.29$ \\
\hline $\mathrm{red} / \mathrm{IK}$ & $1.25 \pm 0.47$ & $(0.67-1.95)$ & $1.07 \pm 0.45$ & $(0.45-1.74)$ & $\mathrm{red} / \mathrm{IK}$ & $1.25 \pm 0.47$ \\
\hline
\end{tabular}


relapse duration for less than 6 months $(71.43 \%$, Z-statistics $=2.5102$, $\mathrm{p}$-value $=0.0121$ ).

The gene expression was analyzed according to the severity of AA. No significant differences were observed between the gene expressions in mild or severe AA, from the lesional or perilesional areas (Tab. 2).

Tab. 2. Mean fold change values for IGF1, IDO1 and red/IK genescomparisons between pairs of data and type of AA

\begin{tabular}{cccc}
\hline Variable & $\begin{array}{c}\text { Severe AA: } \\
\text { m } \pm \text { stdev }\end{array}$ & $\begin{array}{c}\text { Mild AA: } \\
\text { m } \pm \text { stdev }\end{array}$ & $\begin{array}{c}\text { t-value } \\
\text { (p-value) }\end{array}$ \\
\hline $\mathrm{IGF1}^{\mathrm{a}}$ & $2.54 \pm 3.08$ & $2.08 \pm 1.64$ & $0.1923(0.8551)$ \\
$\mathrm{IDO}^{\mathrm{a}}$ & $3.67 \pm 1.19$ & $2.14 \pm 0.99$ & $1.5766(0.1757)$ \\
$\mathrm{red}^{\mathrm{I}} \mathrm{IK}^{\mathrm{a}}$ & $1.17 \pm 0.48$ & $1.45 \pm 0.54$ & $-0.6827(0.5251)$ \\
$\mathrm{IGF}^{\mathrm{b}}$ & $1.80 \pm 1.54$ & $1.90 \pm 0.40$ & $-0.0817(0.9381)$ \\
$\mathrm{IDO}^{\mathrm{b}}$ & $1.67 \pm 0.72$ & $1.93 \pm 0.09$ & $-0.4708(0.6576)$ \\
$\mathrm{red} / \mathrm{IK}^{\mathrm{b}}$ & $0.91 \pm 0.39$ & $1.48 \pm 0.37$ & $-1.7621(0.1383)$ \\
\hline
\end{tabular}

${ }^{\mathrm{a}}$ Lesional vs control; ${ }^{\mathrm{b}}$ Perilesional vs control

Taking into consideration the fact that the duration of AA may also influence cytokines expression, the data were divided according to the length of the relapse: under 6 months and over 6 months. Statistically significant data was obtained for IDO1, mRNA expression being higher at patients with a relapse duration under 6 months in lesional area, $\mathrm{FC}=1.75$ (Fig. 2). The results obtained for IGF1 and $\mathrm{red} / \mathrm{IK}$ did not reach statistical significance.

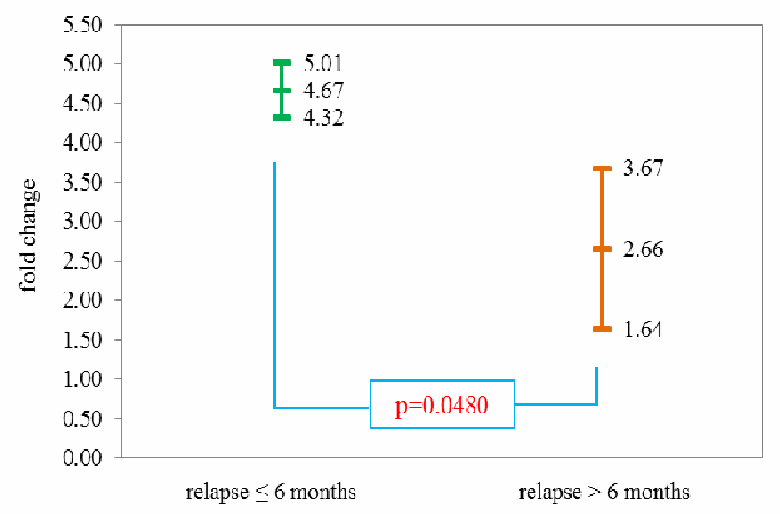

Fig. 2. Mean fold change values for IDO1 in lesional areas ( $95 \%$ CI values of FC) according to relapse duration

\section{Discussions}

In the present study we provide interesting evidence for the involvement of immune privilege factors in AA. Of the three genes that were investigated, all of them had a higher gene expression in the lesional area compared to the perilesional area, however, statistically significant data was obtained for IDO 1.

Indoleamine 2,3-dioxygenase, an IFN-inducible enzyme that suppresses adaptative $\mathrm{T}$-cell immunity by catabolizing the essential amino acid tryptophan from the cellular microenvironment, intervenes in the immunologic balance (Scheler et al., 2007). IDO1 encodes functional IDO protein that regulates $T$ cell responses when expressed in some dendritic cells (Li et al., 2012). Depending on the context, IDO limits immune activator mechanisms, thus preventing a detrimental immune activity, or induces immunosuppression (Hainz et al., 2007). The study performed by Kang (Kang et al., 2010) by carrying through an analysis of 18 genes from biopsy specimens showed, for the first time, that IDO1 was downregulated in AA patients, mostly in the perilesional area. Our results partially confirm this finding, in our study the gene expression of IDO1 being higher in the active edge of the alopecic plaque compared to clinically unaffected scalp. Furthermore, the expression of IDO1 was higher in patients with a relative recent relapse of the disease, under a six month period. We explain these data as an attempt for restoration of the immune privilege in order to stop the progression of the disease and excessive local inflammation.

The involvement of IDO was demonstrated in other autoimmune diseases, such as lichen planus. In this IFNmediated skin condition, IDO is more strongly expressed in lesional skin compared to non-lesional areas (Scheler et al., 2007). Also, as demonstrated on lupus-prone mice, IDO limits the immune system to apoptotic self-antigens and regulates the progression of the autoimmune disease by inhibiting inflammation (Ravishankar et al., 2012). In the case of rheumatoid arthritis, high serum levels of Kyn:Trp that reveal an increased activity of IDO, are positively correlated with the stages of the disease, indicating an increase of IDO with the progression of the illness (Zhu et al., 2006). Our findings are supported by the fact that IDO forms an important counter-regulatory mechanism induced by pro-inflammatory signals, stimulating $\mathrm{T}$ cells also against CMV and tumor antigens (Andersen et al., 2012).

The expression of MHC class II molecules is blocked by several factors, including red/IK cytokine, an antagonist of the IFN gamma induced expression of MHC II (Cao et al., 1997). In the same study performed by Kang (Kang et al., 2010), red/IK was shown to have a lower expression in the perilesional area compared to lesional scalp specimens. The authors suggest that the relative lack of expression of red/IK might allow MHC class II antigen expression and might modulate CD3 + cell development in the hair follicle. In our study, no statistically significant results were obtained, but slightly elevated gene expression of $\mathrm{red} / \mathrm{IK}$ in lesional versus perilesional tissue and slightly higher values of mRNA in mild form of AA in the perilesional area were observed. This data could be explained, considering the immunosuppressive role of red/IK, as an attempt of the hair follicle to restore lost immune privilege. The complex roles of red/IK are just being understood, as it has recently been proven the implication of this molecule in dilated cardiomyopathy (Friedrichs et al., 2009) and in splicing of human type A influenza viruses (Fournier et al., 2014).

On the other hand, the expression of MHC I molecules in human anagen bulbs is influenced by several factors, including $\alpha-\mathrm{MSH}$, TGF- $\beta 1$ and IGF1. Paus et al. (2003) underlined the fact that these molecules downregulate the expression of MHC class I molecules separately and in conjunction, while being part of the restoration mechanism of the immune privilege, a process that is initiated whenever the hair follicle suffers an immune injury. In a previous study (Kang et al., 2010) IGF1 mARN expression was several foldhigher in AA follicles compared to normal hair follicles. Our research supported these results, the fold change (FC) values of IGF1 being higher in the plate-edge area compared to the unaffected area. Even though the complete role of IGF1 is not yet completely understood, it is known the role of IGF1 in hair elongation and maintenance 
266

in the anagen phase: for example, the assessment of IGF1 mARN is being linked with hair shaft elongation after successful radiation therapy (Yoon et al., 2011). Taken together, these data suggest the fact that the increased levels of IGF1 mARN in our patients reflect the tendency of the hair follicle to restore itself.

The research that was performed indicates a different gene expression in lesional and perilesional scalp tissue in alopecia areata patients. The expression of IDO1, IGF1 and $\mathrm{red} / \mathrm{IK}$ was higher in the lesional area, however, it is not proven whether these changes are causative of the clinical expression of the disease or represent a restoration mechanism. A validation of the data on a larger number of patients would also be useful.

In conclusion, our pilot study reveals the differentiated expression of IDO1, red/IK and IGF1 in lesional and perilesional areas, thus supporting the importance of the hair follicle immune privilege in the pathogenesis of AA. Our results underline the importance of the investigated genes, especially IDO1 in the progression of alopecic areas and intrigues for further investigation.

\section{References}

Andersen MH (2012). The specific targeting of immune regulation: T-cell responses against Indoleamine 2,3dioxygenase. Cancer Immunol Immunother 61(8):128997.

Cao LX, Le Bousse-Kerdiles MC, Clay D, Oshevski S, Jasmin C, Krief P (1997). Implication of a new molecule IK in CD34+ hematopoietic progenitor cell proliferation and differentiation. Blood 89(10):3615-23.

Fournier G, Chiang C, Munier S, Tomoiu A, Demeret C, et al. (2014). Recruitment of RED-SMU1 Complex by Influenza A Virus RNA Polymerase to Control Viral mRNA Splicing. PLoS Pathog 10(7):e1004317.doi: 10.1371/journal.ppat.1004164.

Friedrichs F, Zugck C, Rauch GJ, Ivandic B, Weichenhan D, et al. (2009). HBEGF, SRA1, and IK: Three cosegregating genes as determinants of cardiomyopathy. Genome Res $19(3): 395-403$.

Gilhar A (2010). Collapse of immune privilege in alopecia areata: coincidental or substantial? J Invest Dermatol 130(11):2535-7. doi: 10.1038/jid.2010.260.
Hainz U, Jürgens B, Heitger A (2007). The role of indoleamine 2,3-dioxygenase in transplantation. Transpl Int 20(2):118-27.

Ito T, Ito N, Bettermann A, Tokura Y, Takigawa M, Paus R (2004). Collapse and restoration of MHC class -Idependent immune privilege: exploiting the human hair follicle as a model. Am J Pathol 164:623-34.

Ito $T$ (2013). Recent advances in the pathogenesis of autoimmune hair loss disease in alopecia areata. Clin Dev Immunol doi:10.1155/2013/348546.

Kang H, Wu WY, Lo BK, Yu M, Leung G, Shapiro J, McElwee KJ (2010). Hair follicles from alopecia areata patients exhibit alterations in immune privilege-associated gene expression in advance of hair loss. J Invest Dermatol 130(11):2677-80.

Li L, Huang L, Lemos HP, Mautino M, Mellor AL (2012). Altered tryptophan metabolism as a paradigm for good and bad aspects of immune privilege in chronic inflammatory diseases. Front Immunol doi:10.3389/fimmu.2012.00109.

Livak JK, Schmittgen TD (2001). Analysis of Relative Gene Expression Data Using Real-Time Quantitative PCR and the 2-[Delta][Delta]CT Method. Methods,25(4):402-408.

Paus R, Ito N, Takigawa M, Ito T (2003). The hair follicle and immune privilege. J Investig Dermatol Symp Proc 8(2):188-94.

Ravishankar B, Liu H, Shinde R, Chandler P, Baban B, et al. (2012). Tolerance to apoptotic cells is regulated by indoleamine 2,3-dioxygenase. Proc Natl Acad Sci USA 109(10):3909-3914.

Scheler M, Wenzel J, Tüting T, Takikawa O, Bieber T, von Bubnoff D (2007). Indoleamine 2,3-dioxygenase (IDO): the antagonist of type I interferon-driven skin inflammation? Am J Pathol 171(6):1936-1943.

Zhu L, Ji F, Wang Y, Zhang Y, Liu Q, Zhang JZ, et al. (2006). Synovial autoreactive $T$ cells in rheumatoid arthritis resist IDO-mediated inhibition. J Immunol 177:8226-8233.

Yoon SY, Kim KT, Jo SJ, Cho AR, Jeon SI, et al. (2011). Induction of hair growth by insulin-like growth factor-1 in $1,763 \mathrm{MHz}$ radiofrequency-irradiated hair follicle cells. PLoS One 6(12):e28474. doi: 10.1371/ journal.pone.0028474. 